Article

\title{
Walking the First/Last Mile to/from Transit: Placemaking a Key Determinant
}

\author{
Chidambara \\ Department of Physical Planning, School of Planning and Architecture, 110002 New Delhi, India; \\ E-Mail: chidambara17@gmail.com
}

Submitted: 15 February 2019 | Accepted: 12 April 2019 | Published: 30 June 2019

\begin{abstract}
Sustainable mobility concerns have seen cities introducing mass transit systems, but travel choice factors such as accessibility, convenience, comfort and safety cannot be addressed through a transit system alone. A trip made on a transit system requires commuters to utilise more than one mode of transport. Walking is generally the most common transport mode to access and egress transit stops. While there is evidence on the pedestrian environment influencing transit ridership, only a few studies have explored how it affects the share of people who walk to/from transits, especially in the context of the developing world. This article postulates that the pedestrian environment influences users' decision to walk the last mile, substantiating it with the findings of a study of transit users across the metro stations of Delhi, India. A pedestrian environment index is developed by including elements of the built form and activities adjacent to the network of streets, in addition to the pedestrian infrastructure quality. Interestingly, the route environment is found to have a significant and much higher correlation with walk share in contrast to pedestrian infrastructure availability. Within the route environment, the sub-indicator that impacts walk share the most is placemaking. It highlights the significance of planning for an enhanced pedestrian environment in a larger context of the catchment area, in contrast to the current myopic approach of station-centric pedestrian infrastructure provisioning.
\end{abstract}

\section{Keywords}

last mile connectivity; non-motorised transport; pedestrian environment; placemaking; sustainable mobility; walkability

\section{Issue}

This article is part of the issue "Public Space in the New Urban Agenda: Research into Implementation", edited by Michael W. Mehaffy (KTH Royal Institute of Technology, Sweden), Tigran Haas (KTH Royal Institute of Technology, Sweden), and Peter Elmlund (Axel and Margaret Ax:son Johnson Foundation, Sweden).

(C) 2019 by the author; licensee Cogitatio (Lisbon, Portugal). This article is licensed under a Creative Commons Attribution 4.0 International License (CC BY).

\section{Introduction}

One of the ways in which cities are trying to shift the tilt from increasing dependence on private modes of transport to public modes is through the provision of transit systems. Even with a fairly expanded network of state-ofthe-art transit systems criss-crossing the city, it is inconceivable to connect each commuter to his/her doorstep of home, office or elsewhere through it. A trip made on a transit system usually requires the commuter to utilise more than one mode of transport. It is now fairly researched upon that an out-of-transit experience can play an influencing role in travel choice and in the overall appeal of transit systems. Efficient transit connectivity in cities cannot be addressed through a myopic lens of just focusing on building the metro network; it should encompass, within its framework, a very important and often neglected aspect, that of providing good accessibility to the metro. This is where attention is drawn to the issue of last mile connectivity (LMC) of transit systems. In this context, LMC can be defined as both the initial and final leg of delivering connectivity-from origin to transit nodes and from transit nodes to the destination. In this article, LMC signifies both, first and last mile, unless specified. Issues related to LMC can arise from various reasons: lack of adequate walking and cycling infrastructure, unfavourable walking and cycling conditions, service reliability, waiting time and the absence of direct routes 
of feeder bus services, lack of adequate and economical modal interchange options as well as the quality and facilities available at transit nodes (Tay, 2012). Collectively, these become the weak links that can have a ripple effect on the usage of transit as opposed to private modes of transport.

The lack of suitable or adequate last mile (LM) options discourages commuters to shift to public transit. At the same time, it can also affect transit users' LM behaviour, a subject that has not been widely researched. An LM environment that is unconducive may compel transit users to avail of unsustainable transport options for LMC in the form of private modes, such as cars and motorcycles, thereby creating a massive parking demand at transit stations. The space needed for parking and access of private modes of transport adds significantly to the cost of transit stations (Pucher \& Buehler, 2009; Steiner \& Butler, 2006) and attenuates the environmental and traffic benefits of transit service (Bartholomew \& Ewing, 2008). Several factors are known to influence users' LM travel choices-the user's socio-cultural/economic characteristics, trip characteristics and built environment. This article focuses on the pedestrian environment, which can be considered as both a subset and derivative of the built environment.

There is a body of literature establishing the link between transit ridership and the built environment surrounding transit stations (Cervero, 1996; Marshall \& Grady, 2005; Newman \& Kenworthy, 1989; Sung, Choi, Lee, \& Cheon, 2014). The urban fabric surrounding a transit stop is an important decisive factor in whether transit users walk, cycle or take the feeder bus and other paratransit or private modes for their LM commute. Through their Theory of Urban Fabrics, Newman, Kosonen and Kenworthy (2016) have demonstrated that different types of cities have combinations of walking, transit/public transport and automobile/motor car fabrics and that strategic and statutory planning need to have different approaches in each of these. The authors further elaborate that the automobile fabric that overlaps all the walking and transit urban fabrics can even obliterate them, as has been the case in some cities of the US, such as Detroit. Urban streets are a component of the built form. The absence of an adequate walkingfriendly environment can discourage walking even in areas with a walking fabric; conversely, good walking conditions in an automobile fabric can encourage walking. This is especially relevant for LM mode choices where a significant share of LM trips is within walkable distances.

The most common access mode to urban transit, cited in literature, is walk. Cervero (1995) and Loutzenheiser (1997) have concluded that walk access dominates city transit. Olszewski and Wibowo (2005) also observe that walking is the most common and natural transport mode for access to and egress from public transport. They further state that the level of the walking environment may influence public transport usage. In the context of cities in the developing world, re- search points out that the type of mode used for access/egress depends on the distance of the origin point to the transit station (Loutzenheiser, 1997), yet the same studies and other researches (Cervero, 2001; Cervero \& Kockelman, 1997; Ewing, Haliyur, \& Page, 1994; Özbil \& Peponis, 2012; Rodriguez \& Joo, 2004) also indicate that the use of non-motorised transport (NMT), especially walking, for LMC, can greatly be influenced and their catchments enhanced by other factors such as density, land use, street and network design as well as layout and the overall environment.

Although substantial literature exists on linking the surrounding built and the pedestrian environment with transit ridership, there is not much evidence on how these affect the LM travel behaviour of transit users, particularly lacking in the context of cities in the developing world. The difference in LM behaviour patterns of transit users in the developing and the developed world needs to be examined on account of several aspects: the first being the poor condition of walking and cycling infrastructure in the latter; second, the difference in vehicle ownership and income levels and the third, a very important factor, the availability of a wide variety of paratransit modes for both individual hire and shared mobility. In light of these, it is important to study LM trip behaviour (especially with respect to sustainable modes such as walking) in the context of the developing world. It is also believed that cities in the developing world have by and large captive walkers; so the role that the pedestrian environment plays in user behaviour (of walking as LM choice or in terms of enhanced walk catchment sheds) is often underplayed. The key research question that this article addresses is whether the pedestrian environment affects the users' LM trip behaviour and, if yes, which aspect/s of the pedestrian environment are more significant in determining this behaviour. It contends that a more in-depth understanding on the specific aspects of the pedestrian environment that affect LM user behaviour is crucial for long-term sustainable mobility and for better LM planning around station catchments. The article presents the findings of the influence of the pedestrian environment on LM user trip behaviour, which is derived from a broader study on the comprehensive LM planning approach to transits for the city of Delhi, undertaken by the author.

\section{Research Design}

\subsection{Case Study Area Profile}

The Delhi Metro rail has the most expansive network, excluding suburban rail, in the country. Since its operation in 2002, the network has been extended to cover $213 \mathrm{~km}$ of length in seven lines (DMRC, 2018). The average daily ridership of the metro has risen from 0.12 million in 2004-2005 to 2.2 million in 2013-2014, and 2.76 million in 2016-2017. There has been a significant addition in both network length and total ridership between 
the period 2012-2013 and 2016-2017. While the annual growth rate of ridership has seen a decline from $14 \%$ in 2012-2013 and 2013-2014 to 5.8\% in 2015-2016 and 2016-2017, the Compound Annual Growth Rate (CAGR) for this four-year period is $9.3 \%$, which is considerably good. The average daily ridership per $\mathrm{km}$ network length has also grown consistently, from 10,158 to 12,958 , for this four-year period.

The study was carried out at 10 select stations of the Delhi Metro network in the National Capital Region (NCR) of Delhi on two of its busiest lines, namely, the blue and the yellow lines, and a relatively new heritage line (extension of the violet line). Figure 1 shows the location of the case study stations on the network. The network passes through four NCR towns, Noida, Ghaziabad, Gurgaon and Faridabad, of which two stations in Noida have been included.

The criteria used for station selection were station typology (interchange, mid-block and terminal), ridership, density and types of land use in the surrounding vicinity as well as the kinds of LM mode options available to the users. The selected stations included four in the mediumhigh ridership, four in the medium ridership and two in the low ridership categories. Interchange stations were not considered in the selection of stations as the majority of the trips here do not need to access the catchment area outside the stations. The profile of these stations and contextual environment are indicated in Table 1.

Stations with varying types of adjacent land use and activity density in the surrounding context were selected to understand how they affected LM travel behaviour. The population and employment densities around each case study station in Delhi were computed from a transport demand forecast study (RITES, 2010) and classified from low to high activity density (which is reflective of population and employment densities). The land use ranged from purely residential (with varying densities) to a mix of residential-commercialinstitutional (also with varying densities), residentialindustrial-institutional, mixed use (vertical mixing with high activity densities) and heritage.

The metro network of Delhi passes through all the three urban fabrics discussed in the introduction section: the core Central Business District (CBD) areas, characterised by dense mixed use and narrow streets, which qualifies them as having a walking fabric; other medium to high density transit (outer and inner) fabric and low density peripheral areas with an automobile/caroriented urban fabric. Out of the 10 selected case study stations, the context areas of two, namely, Chawri Bazaar (CB) and Red Fort (RF), correspond to the walking fabric. Six stations, Vishwa Vidyalaya (VV), Green Park

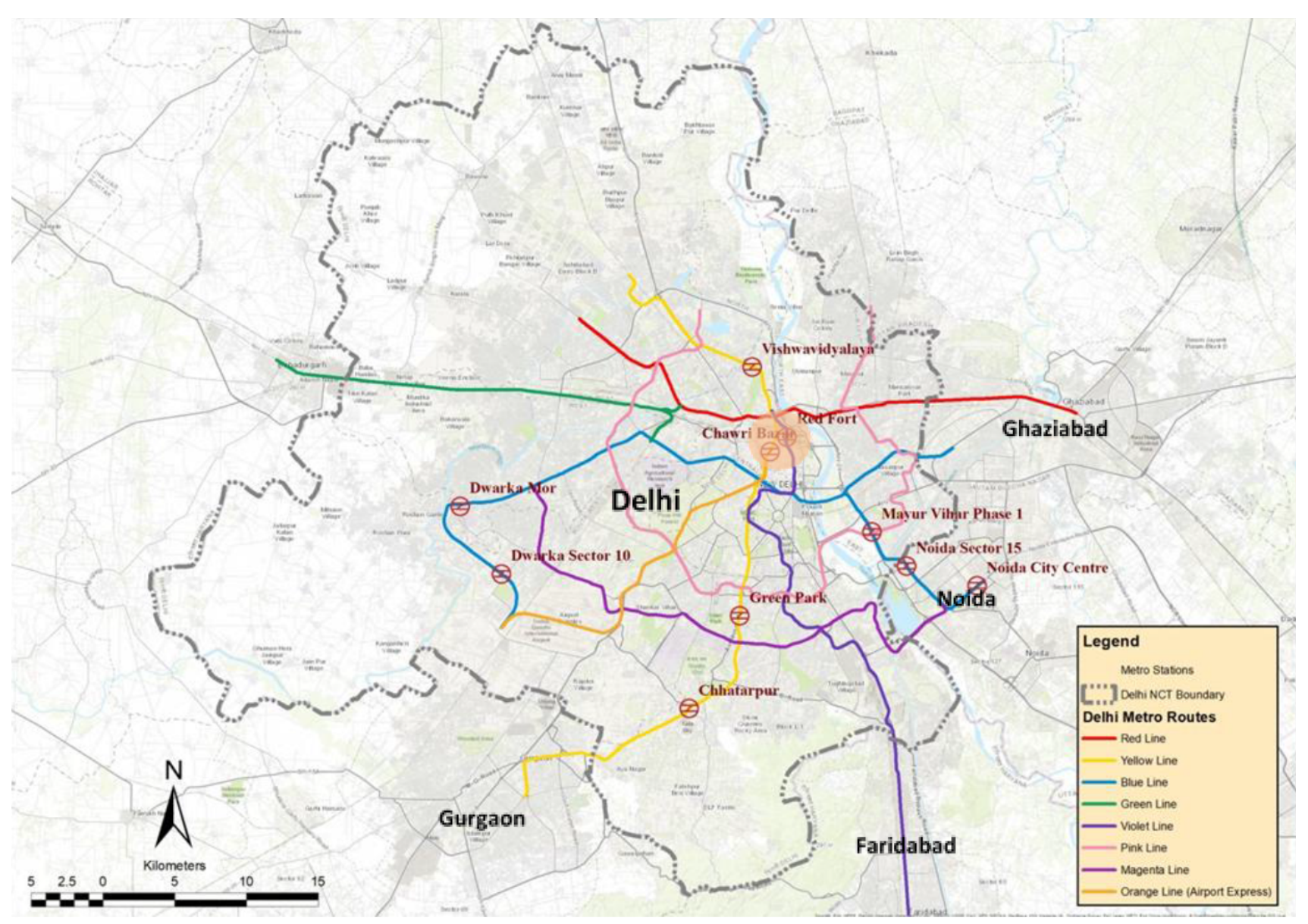

Figure 1. Metro network in Delhi NCR and case study stations. 
Table 1. Profile of case stations, Delhi Metro.

\begin{tabular}{|c|c|c|c|c|}
\hline \multirow[b]{2}{*}{ Station Name } & \multicolumn{2}{|l|}{ Operational } & \multicolumn{2}{|l|}{ Physical Context } \\
\hline & $\begin{array}{l}\text { Avg. Daily Ridership* } \\
\text { (Line Name) }\end{array}$ & Typology & Adjoining Land Use & Activity Density \\
\hline $\begin{array}{l}\text { Chawri Bazar } \\
\text { (CB) }\end{array}$ & $\begin{array}{l}30,798 \\
\text { (Yellow) }\end{array}$ & Mid-block & $\begin{array}{l}\text { Mixed use } \\
\text { Commercial }\end{array}$ & High \\
\hline $\begin{array}{l}\text { Red Fort } \\
\text { (RF) }\end{array}$ & $\begin{array}{l}\text { Low** } \\
\text { (Heritage or Violet line) }\end{array}$ & Mid-block & $\begin{array}{l}\text { Commercial } \\
\text { Mixed use } \\
\text { Heritage }\end{array}$ & High \\
\hline $\begin{array}{l}\text { Vishwavidyalaya } \\
\text { (VV) }\end{array}$ & $\begin{array}{l}23,802 \\
\text { (Yellow) }\end{array}$ & Mid-block & $\begin{array}{l}\text { Residential } \\
\text { Institutional }\end{array}$ & Medium \\
\hline $\begin{array}{l}\text { Noida Sec-15 } \\
\text { (N15) }\end{array}$ & $\begin{array}{l}29,220 \\
\text { (Blue) }\end{array}$ & Mid-block & $\begin{array}{l}\text { Residential } \\
\text { Industrial } \\
\text { Institutional }\end{array}$ & Medium High \\
\hline $\begin{array}{l}\text { Green Park } \\
(\mathrm{GP})\end{array}$ & $\begin{array}{l}27,900 \\
\text { (Yellow) }\end{array}$ & Mid-block & $\begin{array}{l}\text { Residential } \\
\text { Institutional } \\
\text { Commercial }\end{array}$ & Medium High \\
\hline $\begin{array}{l}\text { Dwarka Sec-10 } \\
\text { (D10) }\end{array}$ & $\begin{array}{l}9,761 \\
\text { (Blue) }\end{array}$ & Mid-block & $\begin{array}{l}\text { Residential } \\
\text { Institutional }\end{array}$ & Low \\
\hline $\begin{array}{l}\text { Dwarka Mor } \\
\text { (DM) }\end{array}$ & $\begin{array}{l}42,928 \\
\text { (Blue) }\end{array}$ & Mid-block & Residential & Medium High \\
\hline $\begin{array}{l}\text { Mayur Vihar-I } \\
\text { (MV) }\end{array}$ & $\begin{array}{l}19,413 \\
\text { (Blue) }\end{array}$ & Mid-block & Residential & Medium \\
\hline $\begin{array}{l}\text { Chhatarpur } \\
(\mathrm{CP})\end{array}$ & $\begin{array}{l}36,036 \\
\text { (Yellow) }\end{array}$ & $\begin{array}{l}\text { Mid-block } \\
\text { (last stop in Delhi) }\end{array}$ & Residential & Low \\
\hline $\begin{array}{l}\text { Noida City Centre } \\
\text { (NCC) }\end{array}$ & $\begin{array}{l}37,733 \\
\text { (Blue) }\end{array}$ & Terminal & $\begin{array}{l}\text { Residential } \\
\text { Commercial (partially developed) }\end{array}$ & Medium \\
\hline
\end{tabular}

Notes: * Source: DMRC (2018); ** The station being recently inaugurated, official ridership figure was not available, but falls in low.

(GP), Mayur Vihar-I (MV), Dwarka Mor (DM), Dwarka Sector-10 (D10) and Noida Sector-15 (N15), correspond to the transit fabric, while the last two stations, Noida City Centre (NCC) and Chhatarpur (CP), correspond to the automobile/car fabric. NCC and CP are classified as lying in the automobile fabric on account of being terminal stations or lying in the city periphery along with low/medium population density.

\subsection{Survey Design and Implementation}

Transit commuters were surveyed at 10 selected case study stations through direct questionnaires/interviews, using the pen and paper interview (PAPI) method. In all, 1,000 samples were collected for the 10 stations. The direct questionnaire interview was chosen over other methods, such as online survey, survey through post (self-addressed envelope), because of contextual limitations. First, given the wide diversity of users with respect to literacy and e-literacy levels, language, attitude towards responding on mail, or by post and internet access, there were greater chances of errors on account of not comprehending the question properly, low or poor re- sponse rate and exclusion of certain categories of users. Second, targeting transit users for the specific case study stations would not be possible in an online or web-based survey. Surveys were conducted at entry/exit points of the stations. Although users responded in a much more relaxed and patient manner in on-board surveys, compared to those conducted at entry/exit points, the challenge in an on-board survey was to distinguish the commuters that boarded the train at that particular station. The use of probability methods in this context was not a practical option. In order to avoid selection bias, care was taken to cover all entry/exit points with as many first mile (FM) as LM users and continue till at least some representation of each available LM mode option at the case study station was covered. Surveys were conducted on weekdays, three hours in the morning and three in the evening, which covered the peak ridership hours.

The survey questionnaire included information related to the user's trip characteristics for the undertaken trip, socio-economic characteristics, ranking and rating of indicators considered important for available LM mode choice and the walking environment. The trip characteristics of the FM, the main haul transit trip and 
the LM were covered with respect to mode, time, cost, distance, purpose, frequency and trip-end type at origin/destination. Information on socio-economic characteristics included the user's gender, age, marital status, education level, occupation, individual and household income and personal vehicle availability. For the purpose of this article, the information related to only LM mode choice, trip length and ranking of important indicators for the walking environment are relevant. The LM mode choice of users gave the overall mode share for each station and that was used as the dependent variable (DV) in the models. The ranking of the walkability indicators by the users helped to identify the relative importance placed by them on the different indicators. The sub-indicators 1 to 12 , shown in Table 2, were listed and the users were asked to rank them in order of priority, which they felt was important in deciding whether to walk the FM/LM or use any other mode. These were further used to assign weights to each pedestrian environment indicator in developing the pedestrian environment index (PEI).

Apart from a transit user survey, an assessment of the pedestrian infrastructure and the environment was conducted around case study stations. The audit was carried out for approximately $1 \mathrm{~km}$ length of all major streets (six to eight) adjoining and leading to/from the stations. The components of the pedestrian environment assessed in the audit are discussed in the subsequent section.

\subsection{Pedestrian Environment Indicators}

The understanding of the pedestrian environment has been derived from an exhaustive set of indicators identified through different literature sources discussed in this section. Several studies, although not specifically carried out with the objective of studying LM pedestrian environment, are available. They have incorporated these elements in associating them with walk behaviour or active living. Different studies take different approaches to assessing walkability; some focus on infrastructure provision and quality while others also consider the importance of the overall environment.

Özbil, Yeşiltepe and Argin (2015) have considered aesthetic qualities, signage, sidewalk design, pedestrian crossings/traffic lights, ground floor uses as well as housing plot-level (parcel-level) land use, density and streetlevel topography, street network configurations as well as connectivity measures. Pedestrian safety is another important element in studies on walkability assessments (Boarnet, Anderson, Day, McMillan, \& Alfonzo, 2005; Brown, Werner, Amburgey, \& Szalay, 2007; Gehl, 2011). Other studies have used a wide range of variables such as the dimensions and design of sidewalks, the frontages of retail or the prevailing levels of environmental comfort that may encourage pedestrian movement (Badland \& Schofield, 2005; Ewing \& Handy, 2009), the presence of street crossings and signalisation, attractive landscaping and tree covers (Agrawal, Schlossberg, \& Irvin, 2008; Cao, Mokhtarian, \& Handy, 2007).

The focus on the pedestrian environment has gained further momentum with new urbanism and studies pointing out that improved walking conditions significantly increase the extent of walking and walkingderived activities (Gehl, 2010). The present study thus identifies and includes variables that assess the overall pedestrian environment, featuring elements of the surroundings adjacent to the network of streets and not merely the streets' quality. Most of the elements discussed above were considered while designing the broad indicators and sub-indicators for assessing the pedestrian environment.

The pedestrian environment, thus, in the context of this article, relates to the availability and quality of infrastructure for pedestrian movement between stations and the trip-ends within walking distance. It also considers aspects that are direct derivatives of the physical environment such as the nature of activities, the presence of obscure nooks/stretches, eyes on the street, among others. Direct indicators of the built environment such as land use, densities and network characteristics are not reflected in the pedestrian environment assessment undertaken in this study. These indicators were considered separately as the built environment variables of the broader study from which this article is derived.

The pedestrian environment has been assessed in terms of two broad categories: pedestrian infrastructure and pedestrian route environment. The indicators for each of the two categories and the sub-indicators representing the indicators are given in Table 2. Pedestrian infrastructure was assessed in terms of their availability, condition and quality. The assessment of the route environment was carried out first, in terms of the connectedness (whether the paths connected the metro stations to important destinations of the commuters) and continuity of the paths (whether the paths were continuous and part of an overall network). The second aspect of assessing the route was based on the walking experience for which elements of placemaking were taken into account. These are based on Gehl's human-centric approach to urban design. However, instead of taking exactly the same criteria, indicators that were more suited to the context city and to the objectives of assessing LMC were assimilated from other sources discussed above as well and bunched together. For instance, the aspect of protection, safety and security was considered more important vis-àvis micro-climate. It is also worthwhile to mention here that none of the areas under study have taken conscious placemaking initiatives; however, some of these areas possess inherent traits underlying the principles of placemaking, while others are lacking in them.

Further, each of these sub-indicators has measurable sub-sub-indicators, which were used in designing the audit pro forma. For instance, sub-indicator 1 was measured with respect to the presence (or absence) of footpath, width, kerb height, physical barrier between the 
Table 2. Pedestrian environment indicators.

\begin{tabular}{lll}
\hline Categories & Indicators i & Sub-Indicators \\
\hline Pedestrian Infrastructure & 1 Availability & $\begin{array}{l}\text { Footpath } 1 \\
\text { Crossing } 2 \\
\text { Wayside Amenities } 3\end{array}$ \\
\cline { 2 - 3 } & & Obstructions 4 \\
& 2 Condition \& Quality & Surface 5 \\
Universal Accessibility 6 \\
Route Environment & & Ease of Crossing 7 \\
\hline & & F.P Continuity 8 \\
\cline { 2 - 3 } & 3 Continuity \& Connectivity & Safety \& Security 9 \\
& & Activity \& Liveliness 10 \\
& & Crowdedness 11
\end{tabular}

vehicular and pedestrian path and clear access to path. Similarly, sub-indicator 2 was assessed on the type of crossing facility. Sub-indicator 3 had a list of amenities; sub-indicator 4 included the minimum and maximum effective width available to pedestrians and listed various types of obstructions on the path; sub-indicator 5 comprised the type of paving, maintenance and ramp quality. Sub-indicator 6 , universal accessibility, covered aspects such as the presence of tactile paving and surface quality suitability for prams and wheelchairs. Subindicator 7 was measured through waiting time for crossing and threat from adjacent traffic. Sub-indicator 8 measured footpath continuity in the area, based on the percentage of streets that had continuous paved footpaths. Sub-indicator 9, safety \& security, was assessed in terms of threat from adjacent traffic, presence of obscure areas, presence of nuisance activities, eyes on the street (assessed in terms of presence of active frontage, minimum and maximum front setbacks, direct openings on the streets such as doors/windows) and adequate lighting at night. Sub-indicators 10 and 11 included ratings for activity and liveliness and crowdedness during the day and after dark. Sub-indicator 12, aesthetics, rated the streets in terms of cleanliness, landscaping/visual elements and overall appeal.

Based on the indicators shown in Table 2, a PEI was developed. The weighted factor method has been used to arrive at scores for each indicator of the overall PEls. All the sub-attributes were assigned scores, based on either how well or poorly they met the norms and standards, or on a five-point Likert Scale rating for qualitative sub-attributes. The scores have a maximum scale of five, with one signifying very poor and five representing very good. For instance, the absence of a footpath would denote one and the presence of a footpath without obstructions and a good level of service would denote five. Similarly, an area that is desolate would get a score of one whereas an area that has a lot of activity, but is not overcrowded, would get a score of five. Weights have been assigned to the indicators, based on the users' ranking of indicators for the walk environment. This is derived from the percentage of respondents identifying a particular indicator as most important. $\mathrm{Pi}$ is the weighted score for each indicator and station. The weighted scores are then obtained by multiplying the individual scores (minimum one and maximum five) for each indicator, with their corresponding weights. The individual scores of a particular indicator of a particular station is the average score of all the streets surveyed around it and the average of all the sub-sub-indicators.

The final PEls represent converted percentage scores and have been computed as per Equation 1:

$$
\text { [PEI]s }=\sum_{\mathrm{i}=1}^{4} \mathrm{Pi}[\max : 100 \mathrm{pts}]
$$

Notes: s: station; i: indicator.

The study involved bivariate regression of LM user travel behaviour with the weighted scores of the overall pedestrian environment and each of its components. Bivariate models were considered over multi-variate models because the data set was small (10 points representing values for each of the 10 stations) as each station represented the aggregated values of both the DV and the independent variable (IV). The DV signifying user travel behaviour used in the study is the percentage mode share of walk trips observed at the case study stations and thus has one value for each station. Similarly, each of the IVs represents average scores of all the streets audited around each station. Further, the objective of the study is to see how each aspect of the pedestrian environment independently influences LM walk behaviour and as such a bivariate analysis is considered.

\section{Study Results}

\subsection{Last Mile Mode Shares}

Fifteen different types of mode choices are observed for LM transit users at the case study stations in 
Delhi. These include walk, bicycle, cycle-rickshaw, erickshaw, auto-rickshaw (individual/shared), taxi (individual/shared), feeder bus, city bus, chartered/company bus, car (park and ride/pick or drop) and motorbike (park and ride/pick or drop). However, all these modes are not available or used at all the 10 stations. As seen in Figure 2, walk is the predominant mode for FM or LM (32.5\%), followed by auto-rickshaws (24.6\%), shared auto-rickshaws (12.9\%) and e-rickshaws (11.1\%).

The mode shares of FM or LM trips also vary with respect to the urban fabric typology surrounding the sta- tions. As can be seen from Figure 3, there are some distinct patterns emerging when the stations are grouped in terms of their location on the network. The share of walk trips has the most distinct pattern, with the stations located in the walking fabric (first group )having the highest share, followed by the transit fabric (second group) and the least share observed in the automobile/car fabric (last group). Private mode shares are observed as the maximum in the last group and negligible in the first group of stations. It can also be seen that, within the last group, the station with a higher share of private mode

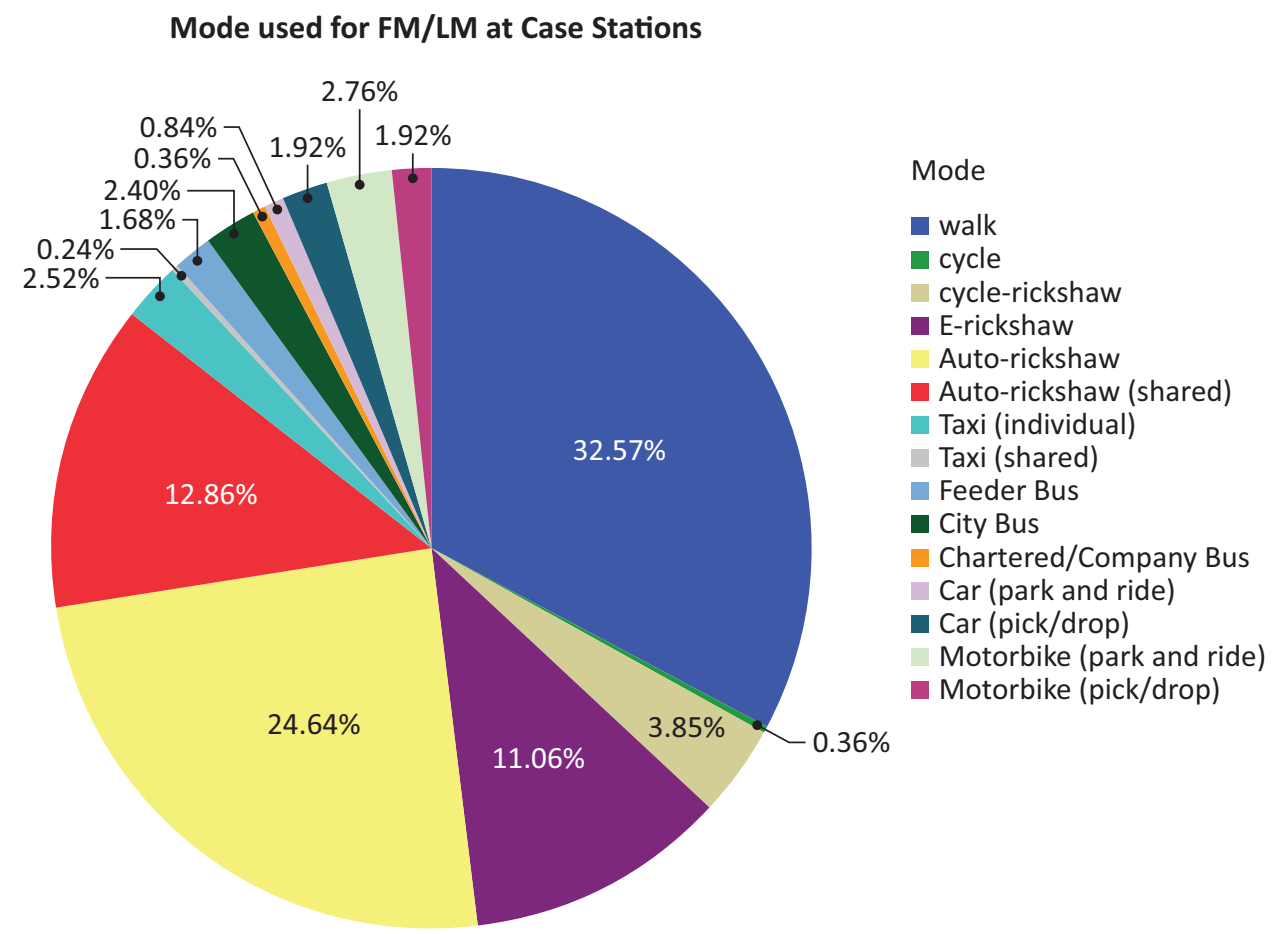

Figure 2. Mode shares for FM or LM.

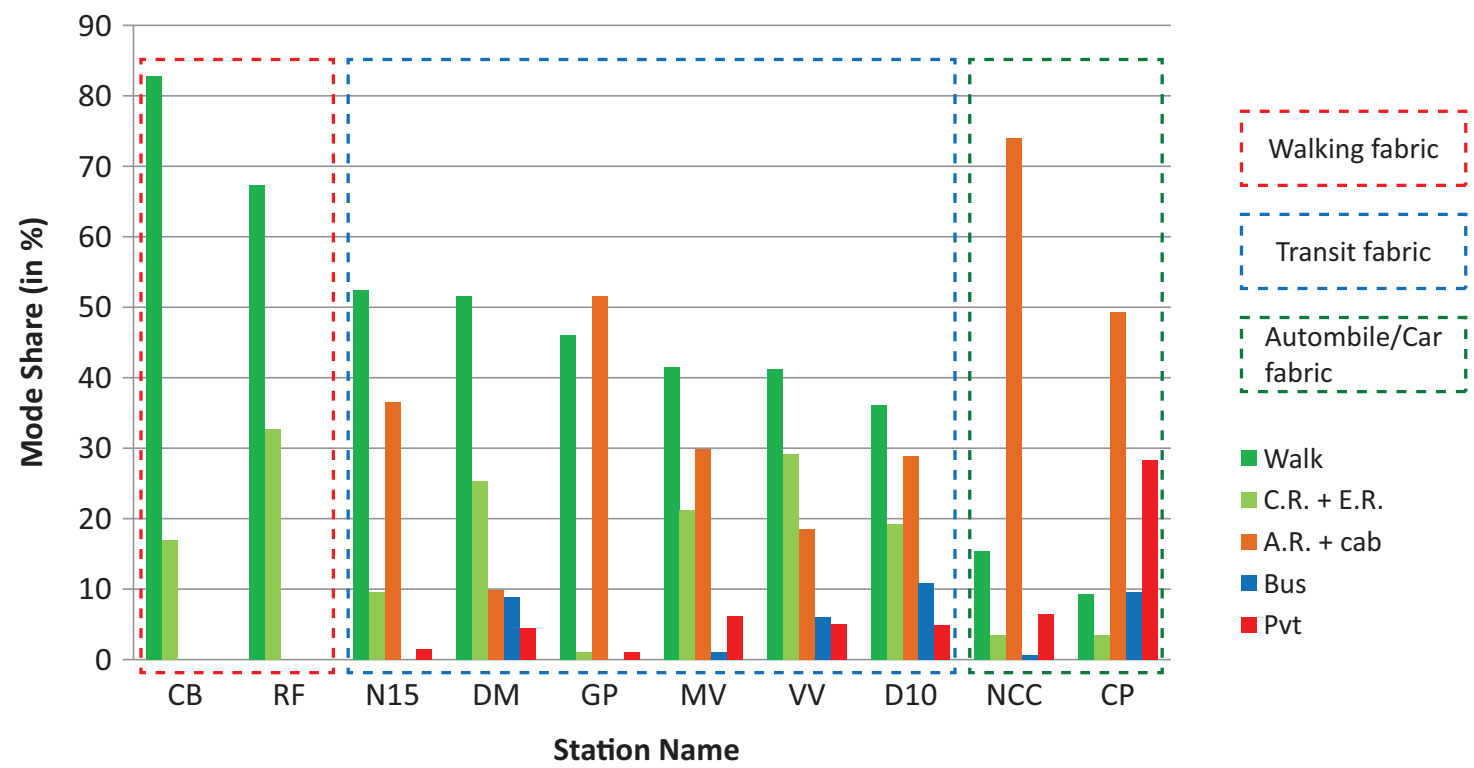

Figure 3. Mode shares for FM or LM for stations in varying urban fabric. Notes: C.R.: Cycle-Rickshaw; E.R.: Electric-Rickshaw; A.R.: Auto-Rickshaw; Pvt: Private Modes. 
trips (CP) has a lower share of walk trips compared to NCC. The stations lying in transit fabric display a mix of various modes of usage for LM and the usage by private modes is lower than that of the stations in the last group. Among the stations lying in the transit fabric, a higher share of walk trips is also observed in those stations that have higher activity density, namely, N15, DM and GP. These findings are indicative of the fact that within the same typology of the urban fabric, there are factors that stimulate a higher share of walk trips.

\subsection{Last Mile Pedestrian Environment}

The scores for all the streets audited around each case study station were averaged out with respect to each of the four main indicators: availability of pedestrian infrastructure, condition and quality, route continuity and connectivity and placemaking. These are shown in Table 3.

The detailed computation of the scores for one station, $C B$, is discussed below.

Pedestrian infrastructure availability [Pi_1 Availability $]_{C B}$ $=$ Average of scores (Footpath availability, Crossings availability, Amenities availability) $=3$

Footpath availability = Average of scores (presence/absence of footpath -4 , footpath width-3, kerb height-3, physical barrier between vehicular and pedestrian path -5 , clear access to path -2 ) $=3.4$

Crossings availability $=$ Average of scores (presence and type as per road type-3) $=3$

Amenities availability $=$ Average of scores (benches-1, trash bins-3, kiosks-5, drinking water -3 , public toilets -1 , streetlights -3 , trees/sheltered walkways -2 ) $=2.6$

Pedestrian infrastructure condition \& quality [Pi_2 condition \& quality $]_{C B}=$ Average of scores (obstructions -2 , surface-2, universal accessibility-1, ease of crossing -4$)=2.2$

Obstructions $=$ Average of scores (minimum and maximum effective width available to pedestrians -2 , nature of obstruction-2) $=2$

Surface $=$ Average of scores (type of paving-3, maintenance -2 , ramps quality -1 ) $=2$

Universal accessibility $=$ Average of scores (presence of tactile paving -1 , surface quality suitability for prams and wheelchairs -1 ) $=1$

Ease of crossing $=$ Average of scores (waiting time for crossing -4 , threat from adjacent traffic -4$)=4$

Route continuity \& connectivity [Pi_3 continuity \& connectivity $]_{C B}=$ Average of scores (continuous and unbroken footpaths-2.6, connection to important destinations in the areas -3 ) $=2.8$

Placemaking [Pi_4 Placemaking $]_{C B}=$ Average of scores (safety \& security -5 , activity and liveliness and crowdedness -5 , aesthetics -3.5 ) $=4.5$

Safety \& security = Average of scores (threat from adjacent traffic-5, presence of obscure areas -5 , presence of nuisance activities -5 , presence of active frontage -5 , minimum and maximum front setbacks -5 , direct openings on the streets such as doors/windows -5 , adequate lighting at night -5$)=5$

Activity \& liveliness and crowdedness = Average of scores (activity \& liveliness and crowdedness-5)

Aesthetics $=$ Average of scores (cleanliness -3.5 , landscaping/visual elements -3 , overall appeal -4$)=3.5$

As discussed in Section 2.3, the weights for each indicator were based on the percentage of respondents identifying that particular indicator as most important in their decision to walk the FM/LM. This resulted in the indicators 'availability of pedestrian infrastructure', 'condition and quality of pedestrian infrastructure', 'route continuity and connectivity' and 'placemaking' receiving weights of $30,15,5$ and 50, respectively. The indicator 'placemaking' got a very high weight since a large number of users identified safety \& security and activity and liveliness as very important, both of which are sub-indicators of 'placemaking' in this study. The scores indicated in Table 3 are multiplied by the corresponding weights of each indicator to obtain station-wise weighted scores for each indicator. The weighted scores, along with the final index, the share of walk trips for LMC and the average trip length of walk trips observed at the respective case study stations are shown in Table 4.

From Table 4 it can be seen that the stations VV (located in a university area) and CB (located in the CBD) top the chart in terms of overall pedestrian environment. It is interesting to note that while VV fares better in terms of

Table 3. Average scores of indicators for all streets surveyed around the case stations.

\begin{tabular}{|c|c|c|c|c|c|c|c|c|c|c|}
\hline Indicators & CB & $\mathbf{R F}$ & N-15 & DM & GP & MV & VW & D10 & NCC & $\mathrm{CP}$ \\
\hline Pi_1Availability & 3 & 2.5 & 3 & 2.5 & 4.2 & 4 & 4.5 & 4 & 3.6 & 3.7 \\
\hline Pi_2Condition \& Quality & 2.2 & 2.1 & 2 & 1.5 & 3 & 3 & 4.5 & 3 & 2.5 & 3.5 \\
\hline Pi_3Continuity \& Connectivity & 2.8 & 3 & 3 & 2.8 & 4 & 3.2 & 5 & 3.8 & 3.5 & 3 \\
\hline Pi_4Placemaking & 4.5 & 4.1 & 3.8 & 4 & 3 & 3 & 3.1 & 2.8 & 2.6 & 2 \\
\hline
\end{tabular}


Table 4. PEI for the case stations.

\begin{tabular}{|c|c|c|c|c|c|c|c|c|c|c|c|c|}
\hline \multirow[t]{3}{*}{ Categories } & \multirow[t]{3}{*}{ Indicators i } & \multirow[t]{3}{*}{ Weight } & CB & $\mathbf{R F}$ & N 15 & DM & GP & MV & VV & D 10 & NCC & CP \\
\hline & & & \multicolumn{10}{|c|}{ Weighted Scores } \\
\hline & & & $\mathrm{Pi}$ & $\mathrm{Pi}$ & $\mathrm{Pi}$ & $\mathrm{Pi}$ & $\mathrm{Pi}$ & $\mathrm{Pi}$ & $\mathrm{Pi}$ & $\mathrm{Pi}$ & $\mathrm{Pi}$ & $\mathrm{Pi}$ \\
\hline \multirow{3}{*}{$\begin{array}{l}\text { Pedestrian } \\
\text { Infrastructure }\end{array}$} & 1. Availability & 30 & 90 & 75 & 90 & 75 & 126 & 120 & 135 & 120 & 108 & 111 \\
\hline & $\begin{array}{l}\text { 2. Condition \& } \\
\text { Quality }\end{array}$ & 15 & 33 & 31.5 & 30 & 22.5 & 45 & 45 & 67.5 & 45 & 37.5 & 52.5 \\
\hline & $\begin{array}{l}\text { Overall Pedestrian } \\
\text { Infrastructure }\end{array}$ & & 123 & 107 & 120 & 97.5 & 171 & 165 & 203 & 165 & 146 & 164 \\
\hline \multirow[t]{3}{*}{$\begin{array}{l}\text { Route } \\
\text { Environment }\end{array}$} & $\begin{array}{l}\text { 3. Continuity \& } \\
\text { Connectivity }\end{array}$ & 5 & 14 & 15 & 15 & 14 & 20 & 16 & 25 & 19 & 17.5 & 15 \\
\hline & 4. Placemaking & 50 & 225 & 205 & 190 & 200 & 150 & 150 & 155 & 140 & 130 & 100 \\
\hline & $\begin{array}{l}\text { Overall Route } \\
\text { Environment }\end{array}$ & & 239 & 220 & 205 & 214 & 170 & 166 & 180 & 159 & 148 & 115 \\
\hline \multicolumn{3}{|c|}{ PEI_overall (Max Score 500) } & 362 & 327 & 325 & 312 & 341 & 331 & 383 & 324 & 293 & 279 \\
\hline \multicolumn{3}{|c|}{$[\mathrm{PEI}] \mathrm{s}=\sum_{1}^{4} \mathrm{Pi}[\max : \mathbf{1 0 0}$ pts$]$} & 72.4 & 65.3 & 65 & 62.3 & 68.2 & 66.2 & 76.5 & 64.8 & 58.6 & 55.7 \\
\hline \multicolumn{3}{|c|}{ Walk share for FM /LM(in \%) } & 82.9 & 67.3 & 52.4 & 51.6 & 46.0 & 41.6 & 41.3 & 36.1 & 15.4 & 9.4 \\
\hline \multicolumn{3}{|c|}{ ATL (Walk) for FM /LM (in km) } & .76 & .84 & .80 & .70 & .76 & 1.18 & .73 & .71 & 1.26 & .43 \\
\hline
\end{tabular}

Notes: The station codes are given in Table 1 earlier; ATL: average trip length.

infrastructure availability, its condition and quality, route continuity and connectivity, $\mathrm{CB}$, despite not faring well in these respects, gets a very good overall score solely on account of its placemaking features. The difference in the pedestrian environment of these stations can be seen in Figure 4 and Figure 5.

Stations such as CB, RF and DM score better in terms of 'placemaking' because the range of activities, eyes on the street and the overall liveliness of the place contribute to a sense of safety \& security and activity \& liveliness to the areas, which are very important for creating conducive walking environments. The first two stations, $\mathrm{CB}$ and $\mathrm{RF}$, are located in the CBD area of the city and have different types of retail and wholesale commercial and tourism activities. The stations lie in the heritage area (Shahjahanabad walled city) of Old Delhi, known for its rich cultural heritage, both of a tangible and intangible nature. It is a high intensity activity area with high resident as well as floating population. The area is lined with narrow winding lanes and closely-packed built mass, originally designed for non-motorised traffic. The many principles underlying 'placemaking' are inherent to the area: mixed use activities, the main street as well as the bylanes with active frontage or buildings with no front setbacks abutting directly onto the streets, a variety of activities taking place on the streets as well as in the buildings along the streets. The station DM, despite not being in the CBD area, also fared well in terms of 'placemaking' because it has a tight built mass with small block lengths, small front setbacks and a host of formal and informal ac- tivities happening along its streets. However, these three stations lose out on the aspect of aesthetics, cleanliness and infrastructure quality.

The last two stations, NCC and CP, which are located in the automobile fabric, also throw some interesting results. A comparison of the walk mode share of these two stations indicates that the station with a higher walk mode share fares better in terms of 'placemaking' and overall route environment, despite scoring lower on the pedestrian infrastructure component.

\subsection{Model Results}

A bi-variate regression analysis of the DV mode share (of walk) was carried out with each of the IVs, which included scores of each of the two broad categories, 'pedestrian infrastructure' and 'route environment', and the scores of the sub-categories, namely, 'availability', 'condition and quality', 'continuity and connectivity' and 'placemaking', respectively. The model results are indicated in Table 5. 'Overall pedestrian environment', 'route environment' and 'placemaking' were observed to be significant, $p<.05$. Although regression with IVs pedestrian infrastructure 'availability', pedestrian infrastructure 'condition and quality', exhibited low/moderate correlation, they were statistically not significant.

There is a moderate $\left(R^{2}=0.40\right)$ relationship between walk share and overall pedestrian environment. Interestingly, route environment is found to be significant with a much higher correlation with walk share in contrast 

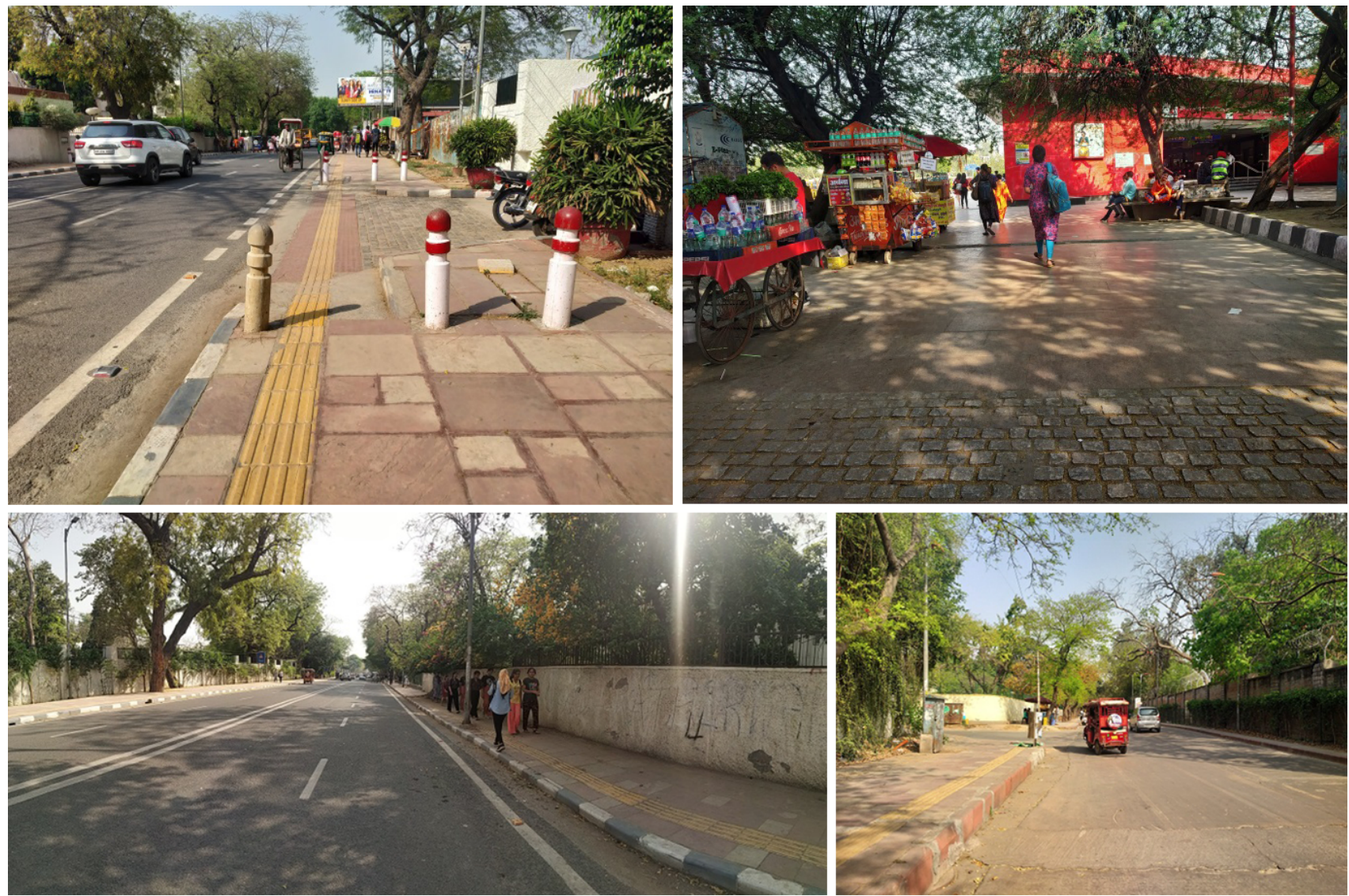

Figure 4. Streets adjoining the VV metro station. Good pedestrian infrastructure, aesthetics and overall cleanliness at the station and in the adjoining streets, but long desolate stretches, lack of visual access from adjoining land uses, large setbacks, long block lengths, very low level of activity and liveliness lower the 'placemaking' scores. Photos credit: Anshika Singh.

Table 5. Models summary.

\begin{tabular}{lllllrr}
\hline Model & DV & IV & $R^{2}$ & $\begin{array}{c}\text { ANOVA } \\
\text { (P value) }\end{array}$ & Coefficients & Intercept \\
\hline 1 & Mode Share (walk) & Overall Pedestrian Environment & 0.4 & .05 & 0.45 & -104 \\
2 & Mode Share (walk) & Route Environment & 0.89 & .000 & 0.55 & -55 \\
3 & Mode Share (walk) & Placemaking & 0.89 & .000 & 0.53 & -42 \\
4 & Mode Share (walk) & Pedestrian Infrastructure & 0.35 & .093 & -0.48 & 112 \\
\hline
\end{tabular}

to pedestrian infrastructure availability. A high linear correlation is observed between walk share and route environment $\left(R^{2}=0.89\right)$. This exhibits the importance which pedestrians in cities of the developing countries attach to the route environment over pedestrian infrastructure availability.

Further, within the route environment the indicator that impacts walk share the most is placemaking. A high linear correlation was observed between walk share and placemaking $\left(R^{2}=.89\right)$. Continuity and connectivity exhibited a low linear relationship and was not statistically significant. The sub-component, pedestrian infrastructure, in fact, exhibited a low negative linear correlation $\left(R^{2}=0.35\right)$ and was also not statistically significant, $p<.1$. The reason for this, probably, could be explained by the fact that stations (selected as case studies) that had a very high share of walk had poor infrastructure, yet their route environment was exceptionally good. These stations also had a relatively higher activity density.

\section{Conclusion}

The study indicates that walk is the predominant mode for LMC to/from transit stations located in walking and transit urban fabrics, with a higher share observed in the former. It is also seen that some stations show a comparatively higher share of walk despite being located in a similar urban fabric. All such stations with higher walk shares, within the same urban fabric, exhibit better performance with respect to placemaking. This is true for stations located across all the three-walk, transit and automobile-urban fabrics. 

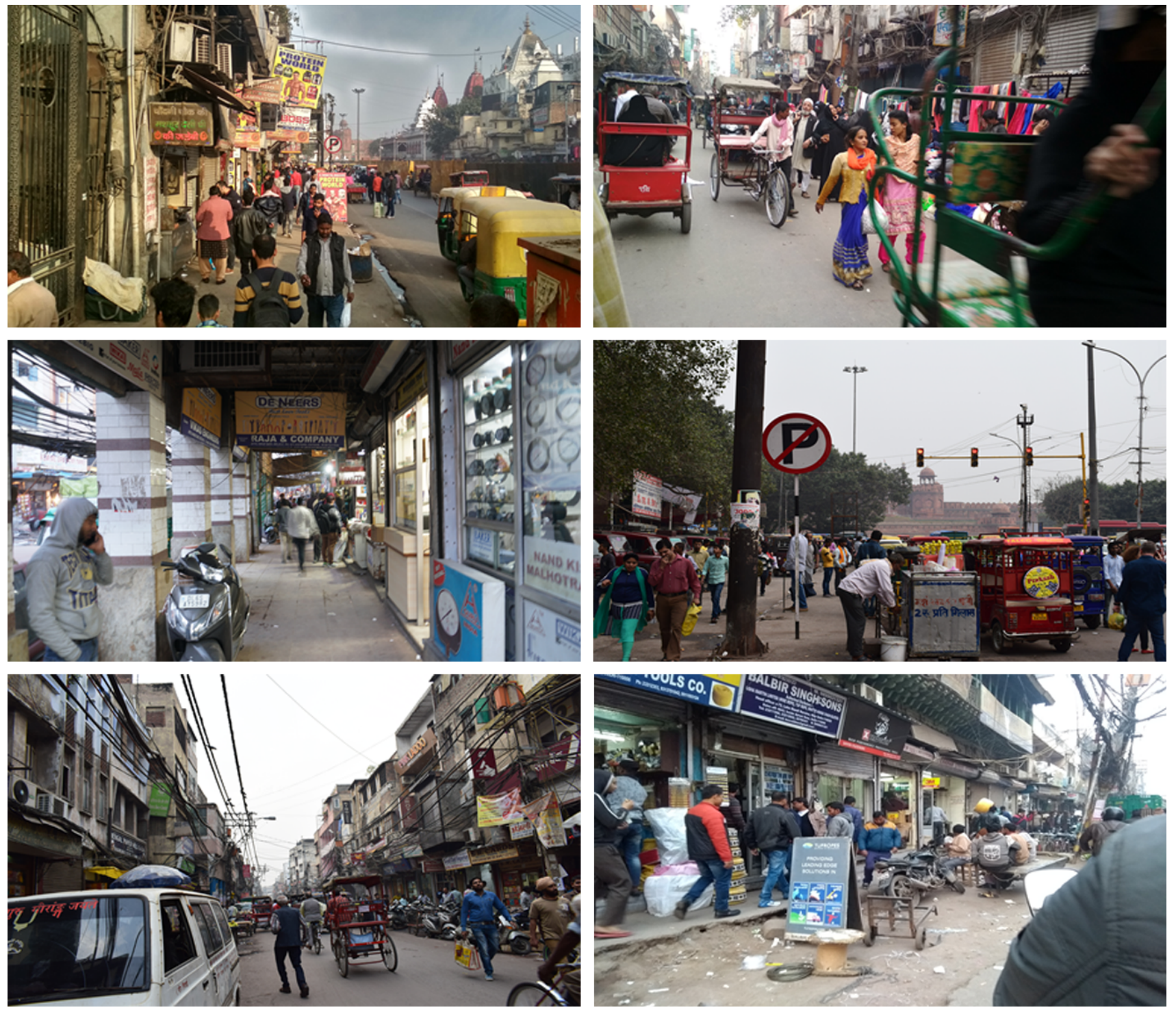

Figure 5. Streets adjoining the CB and RF metro stations. Active frontage, minimum or no front setbacks, a range of continuous formal and informal activities, mixed land use, low vehicular speeds and well-lit pedestrian paths add to the 'placemaking' quality; however, poor aesthetics, infrastructure and lack of cleanliness reduce their overall scores. Photos credit: Nitin Sankhla and author.

The pedestrian environment variables with the most significant influence on walk share were observed to be route quality and its indicator, placemaking. The other indicators of PEI, namely, infrastructure availability, infrastructure condition and quality as well as route continuity and connectivity, exhibited low/moderate correlation and were statistically not significant. Placemaking, which has sub-attributes such as safety \& security, aesthetics, liveliness and activity (a manifestation of activity density) has a significant influence on the user's decision to walk the LM in contrast to just the presence of infrastructure.

The findings of this study are corroborated by other studies undertaken on walkability across various disciplines. Researchers in health and urban design have found pedestrian safety and pleasant conditions to be major factors in determining physical activity levels and in encouraging walking (Boarnet et al., 2005; Brown et al., 2007). The presence of street crossings, attractive landscaping, tree cover and signalisation (Agrawal et al., 2008; Cao et al., 2007), as well as aesthetic or safety features, such as cleanliness, interesting sights and architecture (Appleyard, 1982; Gehl, 2011), have shown higher levels of walking in adults and children.

Further, researches in transportation are also in sync with this study's findings. Shay, Spoon and Khattak (2003) note that measures of accessibility, aesthetics, connectivity and safety are elements that may increase walkability. However, they also observe that the specifics are still open to debate. For instance, while there is general agreement that the provision of sidewalks will improve walkability, there are a host of sidewalk variables that may be important: connections to surrounding destinations, crosswalks, safety features, width, surface quality, lighting and more. 
There is enough conclusive evidence indicating that a better pedestrian environment encourages higher levels of walking. In light of the above findings, it is important that the aspect of placemaking is taken more seriously by planners and metro agencies while drafting their plans for improving the LMC of transit hubs. Most metro stations have considerably good pedestrian infrastructure within the station precinct; however, these need to be extended beyond the station precincts to the catchment areas of the stations. Enhancing walkability around stations needs to be dealt with through a multi-pronged approach. Stations located in the walking fabric, especially in the cities of the developing world, intrinsically possess several elements of placemaking and have a tremendous potential to translate to great streets through focusing on enhancing the aesthetics and infrastructure. However, for stations that are not located in the walking fabric, conscious placemaking interventions become crucial for imparting a better feel of safety and security to walkers. The focus in these areas, even more than, perhaps, infrastructure provision, should be on the application of the concepts of new urbanism for better placemaking.

\section{Acknowledgments}

This article is a part of my Doctoral thesis and I thank all those who have been a part of this journey. I am especially indebted to my PhD supervisor, Prof. Dr. Sanjay Gupta for his guidance and to Prof. Peter Newman who reviewed my doctoral work and gave his invaluable inputs on urban fabric. I also thank my students Anshika Singh and Nitin Sankhla for contributing with photography for the article.

\section{Conflict of Interest}

The author declares no conflict of interest.

\section{References}

Agrawal, A. W., Schlossberg, M., \& Irvin, K. (2008). How far, by which route and why? A spatial analysis of pedestrian preference. Journal of Urban Design, 13, 81-98.

Appleyard, D. (1982). Livable streets. Oakland, CA: University of California Press.

Badland, H., \& Schofield, G. (2005). Transport, urban design, and physical activity: An evidence-based update. Transportation Research Part D: Transport and Environment, 10(3), 177-196.

Bartholomew, K., \& Ewing, R. (2008). Land useTransportation scenarios and future vehicle travel and land consumption: A meta-analysis. Journal of the American Planning Association, 75(1), 13-27.

Boarnet, M. G., Anderson, C. L., Day, K., McMillan, T., \& Alfonzo, M. (2005). Evaluation of the California safe routes to school legislation: Urban form changes and children's active transportation to school. American
Journal of Preventive Medicine, 28, 134-140.

Brown, B. B., Werner, C. M., Amburgey, J. W., \& Szalay, C. (2007). Walkable route perceptions and physical features: Converging evidence for enroute walking experiences. Environment and Behavior, 39(1), 34-61.

Cao, X., Mokhtarian, P. L., \& Handy, S. L. (2007). Do changes in neighborhood characteristics lead to changes in travel behavior? A structural equations modeling approach. Transportation, 34(5), 535-556.

Cervero, R. (1995). Rail access modes and catchment areas for the BART system (BART@20 Working Paper). Berkeley, CA: Institute of Urban and Regional Development, University of California at Berkeley.

Cervero, R. (1996). Mixed land-uses and commuting: Evidence from the American housing survey. Transportation Research A, 30(5), 361-377.

Cervero, R. (2001). Walk-and-ride: Factors influencing pedestrian access to transit. Journal of Public Transportation, 3(4), 1-23.

Cervero, R., \& Kockelman, K. (1997). Travel demand and the 3Ds: Density, diversity, and design. Transportation Research D, 2, 199-219.

DMRC. (2018). Annual report 2017-2018. Delhi Metro Rail Corporation. Retrieved from http:// www.delhimetrorail.com/OtherDocuments/DMRCEnglish-AR-Year-2017-18.pdf

Ewing, R., \& Handy, S. (2009). Measuring the unmeasurable: Urban design qualities related to walkability. Journal of Urban Design, 14(1), 65-84.

Ewing, R., Haliyur, P., \& Page, G. W. (1994). Getting around a traditional city, a suburban planned unit development, and everything in between. Transportation Research Record, 1466, 53-62.

Gehl, J. (2010). Cities for people. Washington, DC: Island Press.

Gehl, J. (2011). Life between buildings: Using public spaces (6th ed.). Washington, DC: Island Press.

Loutzenheiser, D. R. (1997). Pedestrian access to transit: Model of walk trips and their design and urban form determinants around bay area rapid transit stations. Transportation Research Record, 1604(1), 40-49.

Marshall, N., \& Grady, B. (2005). Travel demand modeling for regional visioning scenario analysis. Transportation Research Record: Journal of the Transportation Research Board, 1921, 44-52.

Newman, P., \& Kenworthy, J. (1989). Cities and automobile dependence: A sourcebook. Aldershot: Gower Technical.

Newman, P., Kosonen, L., \& Kenworthy, J. (2016). Theory of urban fabrics: Planning the walking, transit/public transport and automobile/motor car cities for reduced car dependency. Town Planning Review, 87(4), 429-458.

Olszewski, P., \& Wibowo, S. S. (2005). Using equivalent walking distance to assess pedestrian accessibility to transit stations in Singapore. Transportation Research Record: Journal of the Transportation Research Board, 1927, 38-45. 
Özbil, A., \& Peponis, J. (2012). The effects of urban form on walking to transit. In M. Greene, J. Reyes, \& A. Castro (Eds.), Proceedings from the eighth international space syntax symposium (pp. 1-15). Santiago de Chile: Pontificia Universidad Católica. Retrieved from http://sss8.cl/8030.pdf

Özbil, A., Yeşiltepe, D., \& Argin, G. (2015). Modeling walkability: The effects of street design, street-network configuration and land-use on pedestrian movement. A/Z ITU, 12(3), 189-207.

Pucher, J., \& Buehler, R. (2009). Integrating bicycling and public transport in North America. Journal of Public Transportation, 12(3), 79-104.

RITES. (2010). Transport demand forecast study and development of an integrated road cum multi-modal public transport for NCT of Delhi. New Delhi: RITES.

Rodriguez, D. A., \& Joo, J. (2004). The relationship between non-motorized mode choice and the lo- cal physical environment. Transportation Research. Part D: Transport and Environment, 9(2), 151-173.

Shay, E., Spoon, S. C., \& Khattak, A. J. (2003). Walkable environments and walking activity: Final Report. Knoxville, TN: Southeastern Transportation Center, University of Tennessee.

Steiner, F. R., \& Butler, K. S. (2006). Planning and urban design standards. Hoboken, NJ: John Wiley and Sons.

Sung, H., Choi, K., Lee, S., \& Cheon, S. H. (2014). Exploring the impacts of land use by service coverage and station-level accessibility on rail transit ridership. Journal of Transport Geography, 36, 134-140.

Tay, H. (2012). Cycling infrastructure as a first mile solution for mass transit access in Singapore: A study of $M R T$ ridership in Singapore towns (Unpublished Master's Thesis). Massachusetts Institute of Technology, USA. Retrieved from https://dspace.mit.edu/handle/ $1721.1 / 73799$

\section{About the Author}

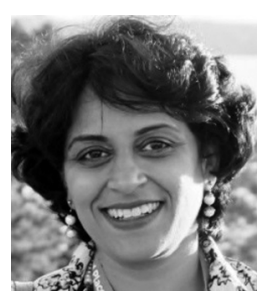

Chidambara (PhD) is an Assistant Professor at the School of Planning and Architecture, New Delhi. She is a transport planner and teaches at undergraduate and graduate planning programmes of the Institute. Prior to this she has worked with planning consultancies, research institutes and NGOs in transport planning, urban development and governance sectors. Her research interests include sustainable mobility planning and policy. In her doctoral work she has developed a comprehensive planning framework for LMC that is responsive to the urban fabric and contexts of transit stations. 